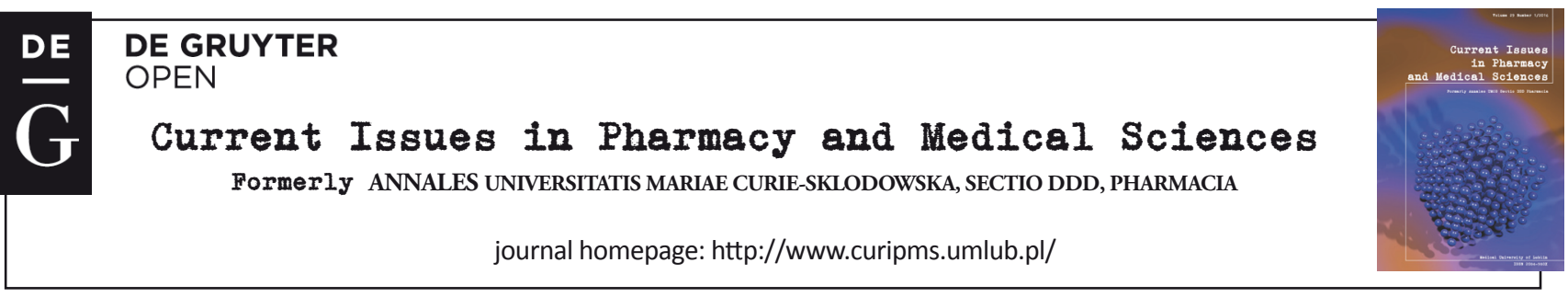

\title{
Using tests and models to assess antidepressant-like activity in rodents
}

\author{
EWA KeDZIERSKA ${ }^{1 *}$, IZABela WaCH ${ }^{2}$ \\ ${ }^{1}$ Chair and Department of Pharmacology and Pharmacodynamics, Medical University of Lublin, Chodzki 4A, 20-093 Lublin, Poland \\ ${ }^{2}$ Student Research Group at the Chair and Department of Pharmacology and Pharmacodynamics, Medical University of Lublin, \\ Chodzki 4A, 20-093 Lublin, Poland
}

\begin{tabular}{l} 
ARTICLE INFO \\
\hline Received 08 February 2016 \\
Accepted 10 March 2016
\end{tabular}

Keywords:

depression,

animal models,

atidepressants.

\begin{abstract}
In today's world, depression is one of the more prevalent forms of mental illness. According to WHO, about $10 \%-30 \%$ of all women and $7 \%-15 \%$ of all men are afflicted by depression at least once in their life-times. Today, depression is assessed to be affecting 350 million people. Regarding this issue, an important challenge for current psychopharmacology is to develop new, more effective pharmacotherapy and to understand the mechanism of action of known antidepressants. Furthermore, there is the necessity to improve the effectiveness of anti-depression treatment by way of bringing about an understanding of the neurobiology of this illness. In achieving these objectives, animal models of depression can be useful. Yet, presently, all available animal models of depression rely on two principles: the actions of known antidepressants or the responses to stress. In this paper, we present an overview of the most widely used animal tests and models that are employed in assessing antidepressant-like activity in rodents. These include amphetamine potentiation, reversal of reserpine action, the forced swimming test, the tail suspension test, learned helplessness, chronic mild stress and social defeat stress. Moreover, the advantages and major drawbacks of each model are also discussed.
\end{abstract}

\section{INTRODUCTION}

Currently, depression is one of the most prevalent psychiatric diseases, and it comes with a complex and heterogeneous pathology. This devastating mental illness is behaviourally characterized by various symptoms, including depressed mood, irritability, low self-esteem, and feeling of hopelessness, feelings of worthlessness and guilt, as well as a decreased ability to concentrate and to think. Other hallmarks of depression are decreased or increased appetite, weight loss or weight gain, insomnia or hypersomnia, low energy, fatigue, or increased agitation and decreased interest in pleasurable stimuli (e.g. sex, food, social interactions). The most dangerous symptoms of depression are recurrent thoughts of death and suicide [22]. This disease also worsens, or even initiates other somatic illnesses, such as cardiovascular [21,9,23], endocrine diseases [27] and cancer [18].

The most widespread accepted theory regarding depression is that of the "Monoamine Hypothesis of Depression" $[6,36]$. This theory establishes that the main reason for

\footnotetext{
* Corresponding author

e-mail: ewa.kedzierska@umlub.pl
}

depression coming about is a deficit in the monoamine neurotransmitters (noradrenaline and serotonin) in the central nervous system. The mechanism of action of most known antidepressants reveals the correctness of this theory, as drugs that increase the levels of these monoamines in the brain are efficacious in the treatment of depression. It should be noted, however, that several other systems are also implicated in the mechanisms underlying the positive effect of antidepressants in treatment of depression. In spite of being clinically effective, most current antidepressant drugs have some imperfections, among these are slow onset and severe side effects [25]. Therefore, the search continues for more promising antidepressant drugs, as well as for better tools for conducting such types of experiments.

Various stressors (particularly, chronic stressors) increase the risk of developing affective disorders in humans. A crucial import into the onset of depression are the alterations in the hypothalamic-pituitary-adrenal (HPA) axis which are activated by acute and chronic stress. As a result of excessive activation of the HPA axis, the level of glucocorticoids in the human system is increased. This causes a cascade of effects, as a sustained elevation of glucocorticoids may 
damage the hippocampal neurons (particularly the CA3 pyramidal neurons) and decrease the expression of brainderived neurotrophic factor (BDNF) $[11,12]$. Additional reasons for depression coming about are abnormalities in the neural circuitry underlying the normal mood.

Epidemiologic data show that about $40 \%-50 \%$ of the risk of depression is genetic, hence, depression can be considered to be a highly heritable disorder [9]. Unfortunately, the specific genes that confer this risk have still not been found. Yet, vulnerability to depression is only partly genetic. Environmental triggers are also very important etiologic factors. Chronic stress, emotional trauma and viral infections may increase vulnerability to depression. Accordingly, depression in most people is activated by interactions between a genetic predisposition and certain encountered environmental factors.

\section{GENERAL CONSIDERATIONS}

The modelling of depression in animals is invaluable, and the perfect animal model can enable a better understanding of the molecular, genetic and epigenetic factors that may evoke depression. Through the use of animal models, we gain an opportunity to generate a better insight into underlying of depression. Moreover, animal models of depression are very useful in discovering new antidepressant medications. There are a lot of difficulties in translating human mental illnesses into relevant tests utilizing rodents. Depression is a heterogeneous disorder, and a full psychiatric syndrome of this illness cannot be mimicked in laboratory animals. Indeed, some symptoms of depression are likely to be purely human features (e.g. low self-esteem, guilt and suicidality). Other symptoms, however, such as helplessness, anhedonia and behavioural despair, can be easily replicated and measured in rodents. To evaluate animal models of depression we employ three main criteria: face validity, construct validity and predictive validity.

Face validity is a consideration of the appropriateness of test or model as a source of data on the compound under investigation. It is understood as a similarity of depression model within the animal subject to the ethology, psychopathology, symptomatology and treatment of the depression disorder in humans, it is measure of external similarity, a kind of analogy. The term construct validity is utilized for the assessment of the empirical and theoretical parallels between the modelled features in animal test subjects and depression in humans, so it is a way of understanding the internal similarity concerning the structure of depression, a kind of homology. The last criterion, predictive validity, refers to the usefulness of the test as employed with animal subjects, in predicting future performance within humans. It describes how good the predictions of how the model in regard to the effectiveness of the researched medications in human subjects will be. Adequate and effective animal models of human depression should fulfill these criteria as much as possible.
All available animal models of depression are based on two principles: the actions of known antidepressants or the responses to stress [41].

Preclinical animal tests and models are aimed at determining the direction of drug action. In such work, the difference between test and model should be noticed. Tests are based on the interactions of potential antidepressants with the tool substances (they are, in fact, behavioural assays for the effects of antidepressants on specific neurotransmitters), and are characterized by simplicity of procedure rules and short duration. In contrast, animal models simulate some aspects of depression. A model, hence, can be defined as a particular state of a non-human organism which mimics some features of human pathology. It provides a certain degree of predictive validity. Animal models of depression are distinguished by greater complexity and longer duration than are tests $[13,42]$.

\section{ANIMAL TESTS BASED ON THE INTERACTION BETWEEN POTENTIAL ANTIDEPRESSANTS AND REFERENCE SUBSTANCES}

\section{Amphetamine potentiation}

Amphetamine potentiation is a classic antidepressant screening test [10]. Indeed, a lot of the effects of amphetamine action (excessive locomotor activity, hypothermia, lower body weight and increase in avoidance reaction) are potentiated by antidepressants. These effects are probably based on the fact that antidepressants impair the metabolism of amphetamine in the liver. As a result, the level of amphetamine concentration in brain is increased (pharmacokinetic interaction) [8]. Other data show that all of antidepressants, apart from mianserin, increase the maximal response to amphetamine (pharmacodynamic interaction) [7]. These dates suggest the utility of the amphetamine potentiation test as a screening test.

Unfortunately, this test is non-specific, sometimes it gives either false negative or false positive results. What is more, newer antidepressants (e.g. mianserin, trazodone) which are structurally dissimilar to tricyclics, do not potentiate the effects of amphetamine action, while other drugs that are not antidepressants also can potentiate this action [41].

\section{Reversal of reserpine action}

This is one of the first tests that have been employed in research on antidepressant-like drugs. Administration of reserpine induces ptosis, catalepsy and hypothermia in rodents. Catalepsy and hypothermia, in turn, can be reversed by compounds which have antidepressant-like activity, among these, the tricyclics and the monoamine oxide inhibitors [6]. However, many new antidepressant drugs (e.g. mianserin, trazodone) do not alter the action of reserpine. In addition, there is also a lot of false positive results seen in this test. Nonetheless, this test sometimes is used, but normally only in connection with other tests and models. 


\section{ANIMAL MODELS BASED ON RESPONSE TO STRESS}

\section{Acute stress models - Predictive models of antidepressant activity}

\section{Forced swimming test}

The forced swimming test (FST) was developed by Porsolt [29-32] so as to examine the antidepressant activity of drugs. FTS is one of the most widely used tests of antidepressant action. Although it works in a subacute condition (30 minutes after drug administration), this test has strong reliability in predicting the therapeutic potential of investigated compounds. In FST, mice or rats are placed into an inescapable cylinder filled with water (Figure 1). In this situation, animals try to leave the cylinder but they cannot escape, so they are eventually induced to displaying a characteristic behaviour of immobility. The test is based on the observation of an immobile posture that is common to rodents. The duration of this immobility in rodents that have been tested after administration of a drug with antidepressant activity is shorter than that seen in a control group. In this test, both groups of drugs, classical as well as atypical antidepressants, are active [30,31].

The Porsolt test has a lot of advantages. Among these are relative simplicity, low costs and the fact that it is a fast and reliable tool for researching potential antidepressant activity (as it has strong predictive validity). This test has a wide range of applications, it is even used as a quick pre-screening test [26]. Unfortunately, the test has poor face and construct validities. Moreover, FST may give false positive results for compounds which increase the general activity (psychostimulants such as caffeine and amphetamine). To verify this, the locomotor activity test is usually employed to control the impact of the investigated compounds on locomotion.
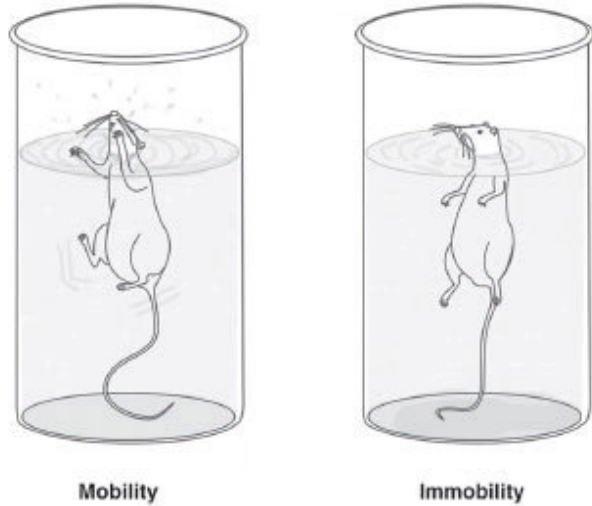

Figure 1. Schematic representation of mobility and immobility in the forced swimming test [1]

\section{Tail suspension test}

The tail suspension test (TST) was introduced by Steru [39] so as to research the potential antidepressant activity of new drugs. This test is based on a theoretical conception that is similar to the conception which is used in the forced swimming test. In this procedure, animals are suspended by their tails (using adhesive tape) to a horizontal bar (Figure 2).
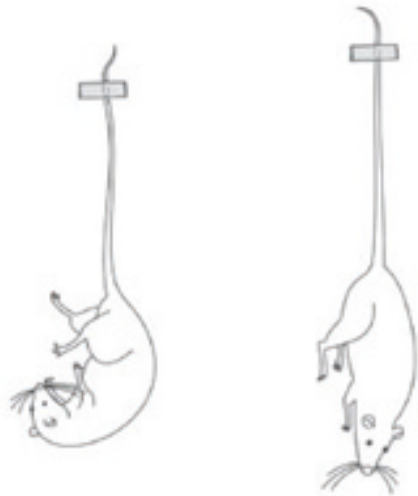

Figure 2. Schematic representation of mobility and immobility in the tail suspension test [1]

At first, the rodents immediately engage in escape-like behaviours, but then, the fact the induced unavoidable and inescapable stress will develop in them, an immobile posture. The test is performed for the duration of six minutes, and the time of immobility is recorded. Clinically effective antidepressants reduce the duration of immobility [42].

One of the advantages of this test is that it can detect a broad spectrum of antidepressants. TST is also inexpensive and unsophisticated. Similarly to FST, one of the drawbacks of TST is the fact that in this test, the acute effect of antidepressant action is measured, which is in contrast with the chronic treatment with the same drug in patients.

\section{Chronic stress models - environmental manipulations}

\section{Learned helplessness}

A well-validated animal model is that of learned helplessness (LH). In this test, animals develop a state of "helplessness" after an exposure to uncontrollable and unescapable stress. Such a depressive-like state in rodents is induced by unpredictable electrical foot-shock stress. After this kind of conditioning, animals are re-exposed to the same shock (but in a different situation when escape is possible) and they still display either increased escape latency or the complete failure to escape [39].

Learned helplessness is induced by several sessions of inescapable shock. Such kinds of uncontrolled, traumatic events result in behavioural and neurovegetative transformations, and the animals develop persistent changes reminiscent of depression, among these, alteration in sleep patterns, reduced body weight, diminished sexual behaviour and alteration in the HPA axis [35].

As of the present, research has indicated that repeated administration of antidepressants decreases the number of animals that show learned helplessness. Moreover, it reduces the latency to escape. In addition, treatment with antidepressants appears to reduce various neurovegetative changes [22].

The main advantages of learned helplessness as a model of depression are its very strong face and predictive validities. This model is considered to be very useful in understanding the depressive symptomatology and pathophysiology of depression in humans. 


\section{Chronic mild stress}

Chronic mild stress (CMS) is one of the chronic stress models which was first developed and described by Katz [15]. This model is based on repeated applications of uncontrollable and unpredictable stress that is interrelated with a quantifiable assay of depression-like behaviour.

The CMS paradigm is composed of relatively continuous exposure of rodents to a wide range of mild stressors. Among these are: minute temperature reductions, changes of cage mates, periods of food and water deprivations, abrupt circadian disruptions and other harmless, but unpredictable manipulations. After an appropriate time period (between 1 and 7 weeks, usually 3 weeks) of exposure to chronic mild stress, certain characteristic changes are observable in the test rodents' behaviour. The most obvious of these is a reduction in sucrose preference ("sucrose preference is calculated as the proportion of sucrose consumption out of total consumption of liquid") [34]. Indeed, sucrose intake is the most commonly used assay for evaluating the impact of CMS in the motivation of rodents towards consuming a dilute solution of sucrose. Moreover, with CMS, the coat state of laboratory animals deteriorates ("coat state is calculated as the sum of the scores areas of the body: head, neck, back, belly, tail, fore-paws, and hind-paws") [38].

Furthermore, CMS is characterized by its remarkable ability to produce a set of behavioural alternations with strong face validity for depression (decreases in sexual, exploratory and locomotor activities and decreased aggressive behaviour). Chronic treatment with antidepressants (including selective serotonin reuptake inhibitors - SSRI, tricyclic antidepressants, serotonin norepinephrine reuptake inhibitors - SNRI, and atypical antidepressants) gradually reverse the reduction in sucrose preference and other symptoms induced by CMS [23].

The advantages of this model are its strong face validity (the behavioural manifestations are similar to the symptoms observed in patients with depression), its construct validity (CMS induces the same pathophysiological changes that occur in patients with depression, such as changes in HPA axis and hippocampal atrophy) and its predictive validity (behavioural changes are reversed by chronic treatment with antidepressants) [24]. Unfortunately, the CMS model has certain disadvantages. It is more expensive than are the other models, and it is difficult to carry out (demanding of space, necessitating extended duration, and intensive labor).

The modification of this paradigm is the chronic unpredictable stress model (CUS). In this model, stronger stress factors are utilized. Among these are electric shock, immersion in cold water, pain caused by tail pressure and immobilization. After three weeks of such stimulations, rodent behaviour is changed. This is seen in a lowered intake of sucrose, a decreased ability to learn, apathy, decreased locomotor activity and prolonged duration of motionless in FST. Herein, the administration of antidepressants prevents the occurrence of behavioural changes during the experiment, but do not reverse already existing changes. This model is consider to be one of the best models for understanding the effects of depression [28].
Social defeat stress

Although both paradigms mentioned above (LH and CMS models) induce long-lasting behavioural, neurobiological and neuroendocrinal alternations, they are of non-social nature. Stressors used in these models are physical, while depression in humans is caused by the majority of stress stimuli that are of social nature $[19,4]$. Hence, investigation of the effects of social stress in experimental animal models is crucial.

The social defeat stress model is based on social and physical interactions between two or more subjects. In this model, social conflict is created between male animals (female rats or mice do not fight each other in residentintruder alteration) [17]. At first, experimental male rodents (intruders) are placed within the territory of aggressive conspecific males (residents). The residents' response is immediate, the intruders are investigated, attacked and defeated by them [13]. After a few minutes of physical interaction, the intruders are separated from the residents and then are influenced by the psychological stress of prolonged "sensory contact" (visual, olfactory and auditory contacts) lasting 24 hours. That situation is repeated for several days.

The experimental animals in the social defeat stress paradigm display reduced social interaction (social avoidance) [16], anhedonia (decreased sucrose preference and sexual activity), decreased exploratory and locomotor activities coupled with physiological, neuroendocrinal and neurobiological consequences of social stress) [2]. Most of these symptoms (especially social avoidance and anhedonia) are long-lasting and can be reversed by chronic, but not acute antidepressant drug administration $[3,14]$. The social defeat stress model is supposed to induce a depressive-like state which may be more relevant to human depression, in comparison with models that utilize acute or severe stressors. Taking under consideration this aspect, the social defeat stress paradigm is generally interpreted as a superior model of human depression. The main disadvantage of this model is the fact that it is very time-absorbing (it requires 20 days of social stress to develop depression). A further drawback is that only male rodents can be used for this model.

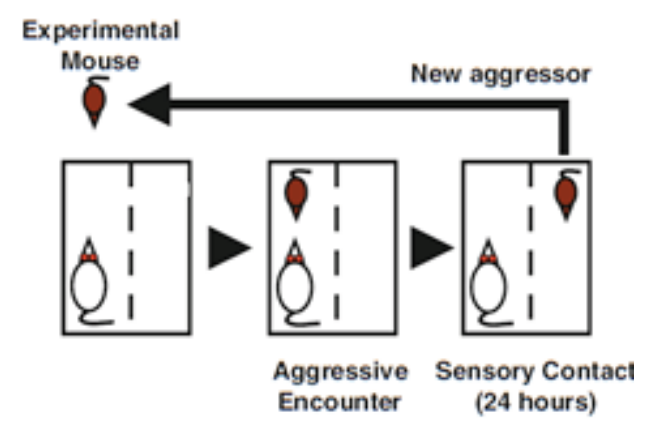

Figure 3. The social defeat model is based on resident-intruder confrontation [40]

\section{CONCLUSION}

Although there are many animal models of depression, there are also a lot of caveats and limitations that constrain 
their usefulness. It is known that translating human affective disorders into relevant tests utilizing rodents is problematic. Nonetheless, scientists still attempt to establish an ideal animal model of depression that displays strong face, construct and predictive validity.

This paper provides a description of the most widely used animal models of depression currently utilized in evaluating anti-depressives and in understanding the depressive state. Moreover, it presents a discussion of the advantages and drawbacks of each model. As revealed, the more rapid and acute antidepressant-responsive assays, such as the FST and TST, are useful tools which allow the quick and economical detection of compounds with potential antidepressant-like activity in which the mechanism of action is similar to the older known drugs. The identification of novel antidepressant mechanisms is made possible by using models that can recreate critical processes operative in depression. Since critical processes are still not deeply known, models that employ stress exposure, time-dependent induction and treatment response are the most appropriate in exploring the mechanisms underlying depression and its treatment.

\section{REFERENCES}

1. Abelaira H.M., Réus G.Z., Quevedo J.: Animal models as tools to study the pathophysiology of depression. Revista Brasileira de Psiquiatria, 35, 2013

2. Avgustinovich D.F., Kovalenko I.L., Kudryavtseva N.N.: A model of anxious depression: persistence of behavioral pathology. Neuroscience and Behavioral Physiology, 35, 917-924, 2005

3. Berton O. et al.: Essential role of BDNF in mesolimbic dopamine pathway in social defeat stress. Science, 311, 864-868, 2006

4. Brown G.W., Prudo R.: Psychiatric disorder in a rural and an urban population: Ethology of depression. Psychological Medicine, 11, 581-599, 1981

5. Coppen A.: The biochemistry of affective disorder. British Journal of Psychiatry, 7, 113, 1237-1264, 1967

6. Costa E., Garattini S., Valzelli L.: Interactions between reserpine, chlorpromazine and imipramine. Experientia, 16, 461-463, 1960

7. Dall'olio R. et al.: Behavioral differentiation between pharmacokinetic and pharmacodynamics components of the interaction of antidepressants or neuroleptics with methamphetamine. Psychopharmacology, 90, 18-23, 1986

8. Everett G.M.: The DOPA response potentiation test and its use in screening for antidepressant drugs. Excerpta Medica, 6, 164-167, 1971

9. Fava M., Kendler K.S.: Major depressive disorder. Neuron, 28, 335-341, 2000

10. Halliwell G., Qunton R.M., Williams F.E.: A comparison of imipramine, chloropromazine and related drugs in various test involving autonomic functions and antagonism of reserpine. $\mathrm{Br}$. J. Pharmacol., 23, 330-350, 1964

11. Holsboer F.: Antidepressant drug discovery in the postgenomic era. World J. Biol. Psychiatry, 2, 165-177, 2001

12. Holsboer F. The corticosteroid receptor hypothesis of depression. Neuropsychopharmacology, 23, 477-501, 2001

13. Hua-Cheng YAN et al.: Behavioral animal models of depression. Neuroscience Bulletin, 26, 327-337, 2010

14. Huhman K.L.: Social conflict models: Can they inform us about human psychopathology? Horm. Behav., 50, 640-646, 2006

15. Katz R.J., Roth K.A., Carroll B.J.: Acute and chronic stress effects on open field activity in the rat: implications for a model of depression. Neuroscience and Biobehavioral Reviews, 5, 247-251, 1981

16. Krishnan V. et al.: Molecular adaptations underlying susceptibility and resistance to social defeat in brain reward regions. Cell, 131, 391-404, 2007

17. Kudryavtseva N.N., Bakshtanovskaya I.V., Koryakina L.A.: Social model of depression in mice of C57BL/6J strain. Pharmacol. Biochem. Behav., 38, 315-320, 1991
18. Lazure K. et al.: Association between depression and survival or disease recurrence in patients with head and neck cancer enrolled in a depression prevention trial. Head Neck-Journal for Sciences Specialties Head Neck, 31, 888-892, 2009

19. Litwack G. (2010). Models of depression. In: Hormones of the limbic system. Duman C.H., p.1-15

20. McKenna M.T et al.: Assessing the burden of disease in the United States using disability-adjusted life years. Journal of Preventive Medicine, 28, 415-423, 2005

21. Musselman D.L., Evans D.L., Nemeroff C.B.: The relationship of depression to cardiovascular disease, epidemiology, biology, and treatment. Archives of General Psychiatry, 55, 580-592, 1998

22. Nestler J. E. et al.: Neurobiology of depression. Neuron, 34, 13-35, 2002

23. Neu P.: Correlation of depression with stroke. Pathophysiological mechanisms. Nervenarzt, 80, 772, 2009

24. Papp M., Moryl E., Wilner P.: Pharmacological validation of the chronic mild stress model of depression. Eur. J. Pharmacol., 296, 129-136, 1996

25. Páez-Pereda M.: New drug targets in the signalling pathways activated by antidepressants. Progress in Neuro-Psychopharmacology and Biological Psychiatry, 29, 1010-1016, 2005

26. Petit-Demouliere B., Chenu F., Bourin M.: Forced swimming test in mice: a review of antidepressant activity. Psychopharmacology, 177, 245-255, 2005

27. Peyrot, M.: Depression: a quiet killer by any name. Diabetes Care, 26, 2952-2953, 2003

28. Popik P.: Metodyka przedklinicznych badań nad depresją i lekami przeciwdepresyjnymi. Depresja i leki przeciwdepresyjne - 10 lat później. Przewłocka B (red.), XIII Zimowa Szkoła Instytutu Farmakologii PAN, 27-44, 1996

29. Porsolt R.D. et al.: Behavioural despair in rats: a new model sensitive to antidepressant treatments. Eur J Pharmacol., 47, 379-91, 1978

30. Porsolt R.D., Bertin A., Jalfre M.: Behavioral despair in mice: a primary screening test for antidepressants. Arch Int Pharmacodyn Ther., 36, 229-327, 1977

31. Porsol R.D., Bertin A. Jalfre M.: Behavioral despair in mice: A primary screening test for antidepressants. Arch. Int. Pharmacodyn. Ther., 229(2), 327-336, 1977

32. Porsolt R.D. Le Pichon M. Jalfre M.: Depression: A new animal model sensitive to antidepressant treatments. Nature, 266(5604), 730-732, 1977

33. Porsolt R.D., Bertin A. Jalfre M.: "Behavioural despair" in rats and mice: Strain differences and the effects of imipramine. Eur. J. Pharmacol., 51(3), 291-294, 1978

34. Pothion S. et al.: Strain differences in sucrose preference and in the consequences of unpredictable chronic mild stress. Behav. Brain. Res., 155, 135-146, 2004

35. Sapolsky R.M.: Glucocorticoids and hippocampal atrophy in neuropsychiatric disorders. Arch. Gen. Psychiatry, 57, 925-935, 2000

36. Schildkraut J.J.: The catecholamine hypothesis of affective disorder: a review of supporting evidence. American Journal of Psychiatry, 122, 1032-1039, 1965

37. Seligman M.E.P., Maier S.F., Peterson Ch. (1995), Learned Helplessness. A Theory for the Age of Personal Control.

38. Stemmelin J, et al.: Implication of beta3-adrenoceptors in the antidepressant-like effects of amibegron using Adrb3 knockout mice in the chronic mild stress. Behav. Brain Res., 206, 310-312, 2010

39. Steru L. et al.: Tail suspension test: A new method for screening antidepressants in mice. Psychopharmacology, 85, 367-370, 1985

40. Vaishnav K., Nestler E. J. (2013). Animal Models of Depression: Molecular Perspectives. In: Molecular and Functional Models in Neuropsychiatry. Hagan J. J. (editor) p.121-148

41. Willner P. (1991). Behavioural models in psychofarmacology. Theoretical, industrial and clinical perspectives. p.91-113

42. Willner P., Mitchell P.J.: The validity of animal models of predisposition to depression. Behav Pharmacol., 13, 169-188, 2002 